\title{
Seepage in Objects: a Primer
}

\author{
Niel Bezrookove
}

January 4th, 2019

\section{Seepage System}

"I've seen Plato's cups and table, but not his cupness and tableness." (109) ${ }^{1}$

Before we begin, we should admit that the purpose of what is to follow is not to argue for a radically new ontology concerning objects. Instead, our hope is to identify the boundaries of what it is we are speaking of when we speak of such things ${ }^{2}$ as an object. An object contains a multiplicity of somethingness, in different modalities. We perceive an object, we describe it using language, we manipulate it physically, and we ascribe uses or ends to them. We understand intuitively that all of these must be related in some sense, as they cohere onto the same object.

I define seepage as a property of improperly bound things, that is our definitions and parameters of a particular thing do not immediately exhaust the properties of that thing. Even if we can define a thing sufficiently to be pragmatic in our usage of language, that does not mean our definition can manage to grasp fully the properties of said thing. In a sudden dramatic event, seepage can occur to something - something novel and informative seeps through the thing and presents itself, a property reveals itself that only now seems inherent to that object. In a sense, the boundary of what constitutes that thing has become permeable while at the same time still being clear. I will call this the permeable structure, a boundary which allows for seepage but still maintains the skeletal shape of the thing we're speaking of.

Let's call that skeletal shape the matrix of the thing. The nature of that matrix is perhaps related to the question of universals - but that is beyond the scope of this paper. Our motivation for denoting this matrix is to allow us to maintain the intuition of some property which connects things together while stressing that things exhibit properties outside of that matrix. The apple as an object is united by a matrix which defines it as an apple, whether the apple has been crushed, rotten, or baked into a pie. Even though properties could seep through the boundaries of that apple to the point of the apple being

\footnotetext{
${ }^{1}$ Herakleitos and Diogenes. Trans. Guy Davenport

${ }^{2}$ I distinguish things from objects to make clear a distinction between a discrete physical form we recognize as an object and the greater 'world' of things which includes objects. This distinction will be of some importance later.
} 
unrecognizable, we still speak of those properties as belonging to the apple we're speaking of - the apple has not lost its appleness.

Of course, the matrix of a thing by definition does not consist of the entirety of the thing. The entirety of an object's properties - how we experience it, how we think of it, its social and cultural significance, its myriad of uses often appears to be larger than our immediate definition of that object. A pupil, Anscombe, raises an important question: "is this a physical property of all objects?" The answer is not immediately obvious. Some objects exhibit much less seepage than others. On one extreme sit objects such as elementary particles, holding a limited set of properties which make its definition rigorous and rigid. On the other extreme sit things such as air, which prima facie seems capable of holding almost any property. This distance between an object's matrix and the entirety of its possible properties as a physical thing is therefore not entirely a matter of perspective or psychology.

We should also note that these properties not only have a system of relations to the frame of the object we're speaking of, but also to other properties. The most obvious system of relations between properties is analogous to some basic operations in set theory. Some properties are physical, like our understanding of a statue as being heavy. In contrast, calling the statue beautiful describes a qualitative experiences involving the object. Some properties can co-exist, many objects can be heavy, tall, and round; some properties cannot, as it would be hard for a lamp to be both on and off. Some properties relate to one another in a manner analogous to a partially ordered set, one property leads to another - for a physical object to possess a change in momentum, something must act upon it. There are also many properties which are dependent on a parent property, as it would be difficult for something to taste bitter if it did not also a taste to begin with. ${ }^{3}$

To say that culture and the skeletal shapes of objects that accompany us in the world share a close historical bond should not be controversial. Heidegger raised this point in his discussion of technology, writing that: " enframing $^{4}$ means the gathering together of that setting-upon which sets upon man, i.e., challenges him forth, to reveal the real, in the mode of ordering, as standing-reserve ${ }^{5}$." (9) ${ }^{6}$ It is the properties that objects reveal which challenges the enterprising human to engage with it. Technology in its most basic form begins with the bringing-forth of properties from the matrix of objects, exploiting the seepage and bringing purpose to what becomes revealed. The discovery of the utility of fire by early Homo was the transformation of a dangerous physical phenomena to an malleable object with a purpose beyond merely existing, and the same process applies to early tools like sticks and stones. The culture of objects, technology's mother, gives matter a frame of existence necessary to allow an existence as an object in our world of things. This culture of objects reveals a

\footnotetext{
${ }^{3}$ The importance of these relations will be appreciated later on in this paper.

${ }^{4}$ Gestell, a gathering together of properties which reveals a way of being seen and understood in the world

${ }^{5}$ An object with properties now revealed by technological activity

${ }^{6}$ Heidegger, M. (1977) The Question Concerning Technology.
} 
tension between our understanding of the object and the physical medium it is carried by, as the mere matrix of an object is insufficient for a culture of objects which holds enframing as one of its modes of existence.

\section{Information is Information}

"Information is information, not matter or energy." (132) ${ }^{7}$

The world which we speak of consists of things ${ }^{8}$, but an object should be defined as more than simply a thing. An object includes properties, as we've described previously, and relations to other objects determined by the possibilities within them. Put more succinctly, "objects contain the possibility of all states of affairs." $(31)^{9}$ Holding our previous discussion of seepage in mind, we can see that the enframing of objects, taking as an example puzzle pieces, allow separate objects to hold relations with each other which reveal unique properties together that their entirely separate matrix forms do not posses. The puzzle pieces do not reveal their representation when they are apart, but begin to put them together with the intent of bringing-forth the representation and you'll see the process of seepage gradually emerge from your activity. This representation is information in the purest sense, a reduction in the uncertainty of our understanding $\mathrm{w} / \mathrm{r} / \mathrm{t}$ the object and its properties ${ }^{10}$.

Information thusly can be defined as a change, not a change in matter or energy, but a change in how objects are organized and what this organization reveals about the objects in question. Any change in organization can reduce the uncertainty $\mathrm{w} / \mathrm{r} / \mathrm{t}$ the properties of the object in question, whether we speak of moving the object (its organization in space) or simply comparing the objects to other objects we can speak of. Our critique of object ontology must thus also address any inattention to the organization of objects, wherever it may occur, as no description of the properties of objects can be complete without addressing information. We can provide here two intuitive examples - water and dominoes.

Water itself is described as an object, but to be more specific what we call water is instead an organization of objects with very specific properties present only due to how said objects behave together. The shape of water, its taste, its appearance are best understood as a property of objects working together rather than the property of one specific $\mathrm{H}_{2} \mathrm{O}$ object. This seems a trivial observation at first, but it is useful when stressing the importance of organization as part of a viable object ontology. Change is how we understand the properties of an object, since the properties which inform us of the object must by our definition

\footnotetext{
${ }^{7}$ Wiener, N. (1948) Cybernetics

${ }^{8}$ Here we could misread Wittgenstein's Tractatus, and proclaim "1.1 the world is the totality of facts, not of things." (29)

${ }^{9}$ Wittgenstein, L. (1922) Tractatus Logico-Philosophicus.

${ }^{10}$ To quote Shannon, "the first defining expression has already been interpreted as the amount of information sent less the uncertainty of what was sent." (22) Shannon, C.E. (1948) A Mathematical Theory of Communication. For a formal definition of uncertainty, refer to Shannon's paper beginning at pg. 10 .
} 
reduce some measure of uncertainty. This change is alluded to by Heidegger, who speaks of "a way of being seen" being revealed and understood in the world. That gathering of properties is its organization, indeed enframing itself should be described as a change in organization - one which transforms water from a collection of objects to a vital resource.

Addressing our second example, dominoes, we mean to say a mechanical computer built from dominoes ${ }^{11}$. This computer carries out an operation through intricately placed dominoes, which perform as transistors do the vital operations required by a computer. This is not done through any inherent properties of dominoes - plastic dominoes cannot perform operations, nor amplify or switch electrical current between terminals. Computation on a domino computer is instead done by arranging the dominoes across a floor, with the appropriate gate operations (AND, XOR, etc.) accomplished using intricate positioning.

Anscombe interjects: "if the computation occurs because of how the dominoes are arranged, does the computer need to be built out of dominoes?" The answer is no. One can find out for themselves that computers can be with some effort built out of many materials, not just silicone or dominoes, and in the case of the computers built within Minecraft ${ }^{12}$ these materials need not even be physical. What is crucial about the computers discussed is not the physical properties of the medium with which they are built, but rather how an informative change in the representation of something ${ }^{13}$ is performed using them. The state of a computer is a property of the computer itself, not of the dominoes which it consists of, yet the entirety of the computer's matter is limited to the dominoes. The computer is a physical object, but one that only exists so long as the dominoes are organized in a manner which allows for computation to seep through the boundaries of its constituent parts. Remove enough dominoes and you'll watch the object itself cease to exist, cease to be informative or revealing, even though the physical properties of the dominoes do not change.

The connection drawn here between water and the domino computer might appear tenuous, as they share few properties and seem to inhabit entirely different worlds. The comparison is meant to illustrate the importance of organization when considering the properties of objects, and to give us two different approaches to understanding what it means for properties to be informative. Anscombe asks: "should we draw the rather obvious conclusion that a lot of objects are actually made of smaller objects?" What is of interest is not that objects consist of smaller objects, but rather that the properties of the objects in question are more than the totality of the properties of its constituent parts. This is true not only when thinking of the chemical composition of substances, but also when considering objects on scales much larger than the basic elements of the periodic table - perhaps a property of all objects no matter the scale.

\footnotetext{
${ }^{11}$ See standupmaths, "The 10,000 Domino Computer."

${ }^{12}$ Please do see: https://minecraft.net/en-us/article/deep-thought

${ }^{13}$ The most important principle of computation is change. If there is no change from state to another, there is no computation.
} 


\section{Percolation}

"The seepage or filtration of a liquid through a porous substance."

We've discussed two aspects of our critique of ontology. We began with a discussion of seepage, the process of properties revealing themselves from the matrix forms of an object. From this discussion came the observation that these properties often have their own system of relations, and we placed this in the context of a culture of objects which engages with this revealing process. What followed was an argument for considering organization as the principle which allows for seepage, which must be understood as an inherently informative process. This should be intuitive - whenever the organization of objects reveals some property, we learn something new and are less uncertain about the object. What motivates this project is a need to move away from fixating on the object and its physical properties when asking what it means for an object to reveal itself; to show "a way of being seen." We wish to consider an object to be a continual process ${ }^{14}$ where the properties represented by the organization of its constituent parts change as they seep beyond its matrix. An object cannot inform us of all of its properties immediately, rather we acquaint ourselves with them ${ }^{15}$ as they are brought forth and becoming enframed as part of how we understand said object. The apple doesn't reveal its taste until we taste it, the water doesn't show its shape until we can see how it moves, etc.

As we admitted from the start, the purpose of this paper was not to establish and defend a new ontology. Our hope is instead to tease out some properties which will allow us to approach object ontology as a process, arguing for an understanding of objects not as rooted in the physical presence of a thing but as a continual interplay between things which realize relations on many levels. Future discussions should address the following ${ }^{16}$ :

- The relationship between our senses and our ability to perceive properties as seeping forth from the boundaries of objects.

- The criteria which distinguish objects exhibiting low seepage from objects exhibiting high seepage.

- Addressing realist versus idealist positions on the nature of relations between and within objects.

- A more in-depth discussion on the process of organization and how properties emerge from that process.

\footnotetext{
${ }^{14}$ The work of A.N. Whitehead is motivated by a similar ambition, and deserves a closer look.

${ }^{15}$ Care must be taken to separate this process from our own process of learning, the relations between objects as we described stand before we become acquainted with them as the domino computer and the water are subject to change whether or not we perceive them.

${ }^{16}$ Many thanks to Magdolin Vartanian and Eric Bracht for their helpful feedback.
} 